INPLASY PROTOCOL

To cite: Ren et al. Efficacy and safety of dapagliflozin in the treatment of chronic heart failure:a systematic review and meta-analysis. Inplasy protocol 2020120071. doi:

10.37766/inplasy2020.12.0071

Received: 11 December 2020

Published: 12 December 2020

Corresponding author:

Guohua Dai

daigh2017@163.com

Author Affiliation:

Shandong University of

Traditional Chinese Medicine

Support: National Key R\&D

Program.

Review Stage at time of this submission: Piloting of the study selection process.

Conflicts of interest:

None.

\section{Efficacy and safety of dapagliflozin in the treatment of chronic heart failure:a systematic review and meta-analysis}

Ren, LL1; Gao, WL2; Guan, $\mathrm{H}^{3}$.

Review question / Objective: The aim of this meta-analysis of randomized controlled trial is to evaluate the efficacy and safety of dapagliflozin in the treatment of chronic heart failure.

Condition being studied: Heart failure is a group of clinical syndromes in which the left ventricular filling or the ability to eject blood is impaired due to abnormal heart structure or (and) function. The main manifestations are dyspnea, fatigue, fluid retention, etc. As the severe and terminal stage of various heart diseases, heart failure has a high recurrence rate and a poor prognosis, which seriously threatens people's physical and mental health. The Framingham Heart Study in the United States shows that the 5-year survival rate for patients with chronic heart failure is only $35 \%$. Therefore, extending the survival period of patients with chronic heart failure, improving the prognosis, and reducing the mortality rate are key issues that need to be resolved. Dapagliflozin belongs to an oral hypoglycemic agent of the SodiumGlucose Transporter 2 Inhibitors. Some studies have shown that such drugs have additional cardiovascular protection, which can reduce the incidence of heart failure hospitalization and other cardiovascular events.

INPLASY registration number: This protocol was registered with the International Platform of Registered Systematic Review and Meta-Analysis Protocols (INPLASY) on 12 December 2020 and was last updated on 12 December 2020 (registration number INPLASY2020120071).

\section{INTRODUCTION}

Review question / Objective: The aim of this meta-analysis of randomized controlled trial is to evaluate the efficacy and safety of dapagliflozin in the treatment of chronic heart failure.
Condition being studied: Heart failure is a group of clinical syndromes in which the left ventricular filling or the ability to eject blood is impaired due to abnormal heart structure or (and) function. The main manifestations are dyspnea, fatigue, fluid retention, etc. As the severe and terminal stage of various heart diseases, heart 
failure has a high recurrence rate and a poor prognosis, which seriously threatens people's physical and mental health. The Framingham Heart Study in the United States shows that the 5-year survival rate for patients with chronic heart failure is only $35 \%$. Therefore, extending the survival period of patients with chronic heart failure, improving the prognosis, and reducing the mortality rate are key issues that need to be resolved. Dapagliflozin belongs to an oral hypoglycemic agent of the Sodium-Glucose Transporter 2 Inhibitors. Some studies have shown that such drugs have additional cardiovascular protection, which can reduce the incidence of heart failure hospitalization and other cardiovascular events.

\section{METHODS}

Participant or population: Patients with chronic heart failure (as diagnosed by a clinician, or using any recognized diagnostic criteria) will be included.

Intervention: Dapagliflozin is the main intervention.The treatment group was given Dapagliflozin $(5-10 \mathrm{mg})$ on the basis of conventional treatment of chronic heart failure. Conventional treatments mainly include diuretics, ACEI, ARB, $\beta$-receptor blockers, aldosterone receptor antagonists, ivabradine, digitalis drugs, vasodilators, inotropic drugs, anticoagulants, etc.

Comparator: The control group was only given conventional treatment of chronic heart failure, or the same dose of placebo was given on the basis of conventional treatment of chronic heart failure.

Study designs to be included: Randomized controlled trial(RCTs) will be included irrespective of language or publication type.

Eligibility criteria: Eligibility criteria under the guidance of the PICOS principle include the following.Participants. Patients with chronic heart failure (as diagnosed by a clinician, or using any recognized diagnostic criteria) will be included.Patients who have received acute heart failure, or patients with severe liver and kidney, or blood diseases, or severe mental health conditions, malignant tumors, or other uncontrolled systemic diseases are excluded.No restrictions are imposed on nationality, age, gender, and race.Interventions and comparators. The treatment group was given Dapagliflozin $(5-10 \mathrm{mg})$ on the basis of conventional treatment of chronic heart failure. The control group was only given conventional treatment of chronic heart failure,or the same dose of placebo was given on the basis of conventional treatment of chronic heart failure. Conventional treatments mainly include diuretics, ACEI, ARB, $\beta$-receptor blockers, aldosterone receptor antagonists, ivabradine, digitalis drugs, vasodilators, inotropic drugs, anticoagulants, etc.Outcomes.The primary efficacy outcome indicators include mortality and heart failure rehospitalization rate; the secondary outcome indicators include New York Heart Association classification (NYHA classification), 6-minute walk test, left ventricular ejection fraction (LVEF), brain natriuretic peptide (BNP)/N-terminal B-type natriuretic peptide precursor (NTproBNP), quality of life (QOL), etc.; the safety indicators include hypovolemia, hypoglycemia, kidney damage and other adverse reactions.Study of type. Randomized controlled trial(RCTs) will be included irrespective of language or publication type.

Information sources: Eight electronic databases( PubMed, EMBASE, the Cochrane Library, Web of Science, China National Knowledge Infrastructure, Wanfang Data, Chinese Scientific Journal Database, Chinses Biomedical Literature Database) will be searched. In addition to a comprehensive search of the databases, tracking of references, and retrieving through search engines are also carried out.For trials where the original paper is incomplete or has not yet been published, 
all relevant authors will be contacted to obtain the required data.

Main outcome(s): The primary efficacy outcome indicators include mortality and heart failure rehospitalization rate; the secondary outcome indicators include New York Heart Association classification (NYHA classification), 6-minute walk test, left ventricular ejection fraction (LVEF), brain natriuretic peptide (BNP)/N-terminal B-type natriuretic peptide precursor (NTproBNP), quality of life (QOL), etc; the safety indicators include hypovolemia, hypoglycemia, kidney damage and other adverse reactions.Study of type.

Quality assessment / Risk of bias analysis: Two reviewers will independently assesses the quality of the selected studies according to the Cochrane Collaboration's tool for randomized controlled trials. When assessing the quality of the selected research, if there is a disagreement between two reviewers, we will try to reach a consensus. However, if a consensus cannot be reached, the third reviewer resolves the issue.According to Cochrane Handbook V.5.2.0, the following characteristics will be evaluated: Random sequence generation (selection Bias) ,Allocation concealment (selection bias) ,Blinding of participants and personnel (performance bias) ,Incomplete outcome data (attrition bias) ,Selective reporting (reporting bias), Other biases. characteristics of each Item will be evaluated in three categories: Low risk of bias, unclear bias and high risk of bias. Results from these questions will be graphed and assessed using Review Manager 5.3.

Strategy of data synthesis: The metaanalysis was performed with Review Manager 5.3 and STATA 14.2 software. For binary variables, we will calculate a summary estimate with $95 \%$ confidence interval (Crls) OR value; for continuous variables, we will calculate a summary estimate of standardized mean difference (SMD) with $95 \%$ confidence interval value(Crls).Between- study heterogeneity will be assessed using the $Q$ statistic test method and 12 statistic method.When the $Q$ statistic corresponds to $P \leq 0.10$ or $12>50 \%$, it indicates that there is heterogeneity, and the random effect model is used to merge the effect size; otherwise, the fixed effect model is used. Results will be assessed using forest plots. An influence analysis will be performed to ascertain the results of the meta-analysis by excluding each of the individual studies. Publication bias will be assessed by a funnel plot for meta-analysis and quantified by the Egger method.

Subgroup analysis: If there is a high degree of heterogeneity in the study, we conduct a subgroup analysis to explore age, gender, ect. At the same time, in order to understand whether the left ventricular ejection fraction will affect the therapeutic effect of dapagliflozin, it will be used for chronic heart failure patients with LVEF $<40 \%$ and chronic heart failure with unknown LVEF the mortality rate and heart failure rehospitalization rate were analyzed by subgroups. In order to understand whether patients with heart failure and type 2 diabetes affect the therapeutic effect of dapagliflozin, the mortality rate and heart failure rehospitalization rate will be subanalyzed on whether patients with heart failure have type 2 diabetes.

Sensibility analysis: This study will use the method of eliminating each study one by one for sensitivity analysis. If the effective indicators of dapagliflozin in the treatment of chronic heart failure (the mortality rate and heart failure rehospitalization rate) have not changed significantly, it indicates that the study is stable. On the contrary, according to the specific situation, lowquality research is excluded.

Country(ies) involved: China.

Keywords: Chronic heart failure; dapagliflozin; systematic review;metaanalysis.

Contributions of each author:

Author 1 - Lili Ren.

Author 2 - Wulin Gao.

Author 3 - Hui Guan. 